C. Sabbah

Nagoya Math. J.

Vol. 141 (1996), 107-124

\title{
ON THE COMPARISON THEOREM FOR ELEMENTARY IRREGULAR $\mathscr{D}$-MODULES
}

\author{
CLAUDE SABBAH
}

\section{Introduction}

Let $U$ be a smooth quasi-projective variety over $\mathbf{C}$ and let $f$ be a regular function on $U$. Let $\mathscr{D}_{U}$ be the sheaf of algebraic differential operators on $U$ and let $\mathcal{M}$ be a regular holonomic $\mathscr{D}_{U}$-module: here, regular means that there exists some smooth compactification $X$ of $U$ and some extension of $\mathcal{M}$ as a $\mathscr{D}_{X}$-module which is regular holonomic on $X$ (one also may avoid the use of a smooth compactification to define regularity, see [17]).

Let $\mathcal{M}_{f}$ be the $\mathscr{D}_{U}$-module obtained from $\mathcal{M}$ by twisting by $e^{f}$. By definition, $\mathcal{M}_{f}$ is equal to $\mathcal{M}$ as an $\mathscr{O}_{U}$-module; the operator $\nabla_{f}: \mathcal{M}_{f} \rightarrow \Omega_{U}^{1} \otimes_{\mathscr{O}_{U}} \mathcal{M}_{f}$ is equal to $e^{-f} \nabla e^{f}$, where $\nabla$ is the operator $\mathcal{M} \rightarrow \Omega_{U}^{1} \otimes_{\mathscr{O}_{U}} \mathcal{M}$ given by the $\mathscr{D}_{U}$-module structure; we have $\nabla_{f}^{2}=0$ because $\nabla^{2}=0$ and this defines a $\mathscr{D}_{U}$-module structure on $M_{f}$.

Let $\operatorname{DR}(\mathcal{N})$ be the algebraic de Rham complex of the holonomic $\mathscr{D}_{U}$-module $\mathcal{N}$ :

$$
\operatorname{DR}(\mathcal{N})=\left\{0 \rightarrow \mathcal{N} \stackrel{\nabla}{\rightarrow} \Omega_{U}^{1} \otimes_{\mathscr{O}_{U}} \mathcal{N} \stackrel{\nabla}{\rightarrow} \Omega_{U}^{2} \otimes_{\mathscr{O}_{U}} \mathcal{N} \stackrel{\nabla}{\rightarrow} \cdots\right\}
$$

(it is now usual to consider that the term corresponding to $\Omega^{\operatorname{dim} U}$ is in degree 0 , but it will not matter here and we shall not shift this complex). We shall give a formula for the hypercohomology of $\operatorname{DR}\left(M_{f}\right)$, i.e. the cohomology of the complex $R \Gamma\left(U, \mathrm{DR}\left(M_{f}\right)\right)$. If $U$ is affine, this is the cohomology of the complex $\operatorname{DR}\left(M_{f}(U)\right)$ of global sections over $U$.

This result was conjectured in [1] in a particular case, where $U$ is the complement of an arrangement of hyperplanes in general position in $\mathbf{C}^{l}$ and $\mathcal{M}$ is a rank one locally free $\mathscr{O}_{U}$-module.

In fact, the global comparison theorem we give is essentially equivalent to the one given in [8] (see also [15] and [22]).

We shall use this result to obtain vanishing theorems of the type given in [1]

Received October 18, 1994. 
under weaker assumptions on the arrangement or on the regular function.

We also prove a local version of the comparison theorem (see $\S 0$ for all the following undefined notations): in the algebraic case for instance, if $X$ is a compactification of $U$ on which $f$ extends as a function $F: X \rightarrow \mathbf{P}^{1}$, and if $\eta$ denotes the inclusion $U \hookrightarrow X$, we shall give a topological formula to compute the analytic (hence algebraic by GAGA) de Rham complex of the $\mathscr{D}_{X}$-module $\eta_{+} \mathcal{M}_{f}$.

We deduce from this result that the irregularity complex of $\mathcal{M}_{f}$ (see [18]), which is the cone of

$$
\mathrm{DR}^{\mathrm{an}}\left(\eta_{+} \mathcal{M}_{f}\right) \rightarrow R \eta_{*} \mathrm{DR}^{\mathrm{an}}\left(\mathcal{M}_{f}\right)
$$

has the same characteristic function as the nearby cycle complex $\phi_{1 / F}\left(R \eta_{*} \mathrm{DR}^{\text {an }} \mathcal{M}\right)$.

Because the characteristic cycle of $\eta_{+} \mathcal{M}_{f}$ can be computed only in terms of the characteristic function of the complex $\mathrm{DR}^{\mathrm{an}}\left(\eta_{+} \mathcal{M}\right)$, this result corroborates the computation in [4].

The results proved in this article are more or less known to specialists, but do not seem to exist with enough generality in the literature.

We shall assume that the reader is familiar with the theories of algebraic $\mathscr{D}$-modules, derived categories and perverse sheaves. One is referred to [2], [16] and [10] for more details on these. However we shall recall below some known facts in these theories and give some more precise references.

\section{A quick trip through the theory of holonomic $\mathscr{D}$-modules}

0.1. We shall denote $\mathscr{D}_{X}$ the sheaf of algebraic differential operators on a smooth algebraic variety over $\mathbf{C}$ (see [3, p. 207] or [16, p. 24]). By a $\mathscr{D}_{X}$-module we will mean a coherent left $\mathscr{D}_{X}$-module.

0.2. Let $\varphi: Y \rightarrow X$ be a proper morphism between smooth algebraic varieties over $\mathbf{C}$. We denote $\varphi_{+}$the direct image of $\mathscr{D}_{Y}$-modules (see [3, p. 240] or [16, p. 61]). If $\mathcal{M}$ is a $\mathscr{D}_{Y}$-module, $\varphi_{+} \mathcal{M}$ is a bounded complex, the cohomology of which is made of $\mathscr{D}_{X}$-modules (see [3, p. 275] or [16, p. 75]).

When $\varphi$ is not proper, the previous result is not true in general, but remains true when applied to holonomic $\mathscr{D}_{Y}$-modules. In this case holonomicity is preserved by $\varphi_{+}$(see [3, p. 292] or [16, p. 77]).

0.3. The dual $D_{X} \mathcal{M}$ of a holonomic $\mathscr{D}_{X}$-module $\mathcal{M}$ is also a holonomic $\mathscr{D}_{X}$-module: in this case, $\mathscr{D}_{X} \mathcal{M}$ is the left $\mathscr{D}_{X}$-module associated with the right 
$\mathscr{D}_{X}$-module $\mathscr{E} x t_{\mathscr{D}_{X}}^{\mathrm{dim} X}\left(\mathcal{M}, \mathscr{D}_{X}\right)$

The duality functor is in fact defined at the level of complexes. Moreover there is a biduality theorem $D_{X}\left(D_{X} \mathcal{M}\right) \simeq \mathcal{M}$ (see [3, p. 277] or [16, p. 40]).

When $\varphi: Y \rightarrow X$ is proper, the duality functor commutes with direct images (up to a shift of complexes depending on the convention), namely $\varphi_{+} D_{Y}=D_{X} \varphi_{+}$ (see [3, p. 278] or [16, p. 74]).

However, when $\varphi$ is not proper, it will not commute in general and we define a new functor (we must restrict here to holonomic objects) $\varphi_{\dagger}=D_{X} \varphi_{+} D_{Y}$.

0.4. We will consider $\varphi_{\dagger}$ in the case where $\varphi=\eta: U \hookrightarrow X$ is the inclusion of a Zariski open set such that $X-U$ is a divisor $D$. If $\mathcal{M}$ is a holonomic $\mathscr{D}_{U}$-module, $\eta_{+} \mathcal{M}$ is the only holonomic $\mathscr{D}_{X}$-module such that

(1) $\eta_{+} M_{\mid U}=\mathcal{M}$

(2) if $h$ is a local equation for $D$, multiplication by $h$ is bijective on $\eta_{+} M$ (i.e. multiplication by $h^{-1}$ is defined on $\eta_{+} \mathcal{M}$ ).

Using (0.3) we can also define $\eta_{+} M$ but its characteristic properties are more difficult to state directly.

0.5. Given a holonomic $\mathscr{D}_{X}$-module $\mathcal{M}$, the constructibility theorem of Kashiwara (see [9] and also [19] or [20]) asserts that the analytic de Rham complex $\mathrm{DR}^{\text {an }} \mathcal{M}$ (defined as the algebraic one (*) using holomorphic forms) is a bounded complex with constructible cohomology on $X$ (in other words a constructible complex). We will denote ${ }^{p} \mathrm{DR}=\mathrm{DR}[\operatorname{dim} X],{ }^{p} \mathrm{DR}^{\mathrm{an}}=\mathrm{DR}^{\mathrm{an}}[\operatorname{dim} X]$.

For $\varphi: Y \rightarrow X$ as above and $\mathcal{M}$ a holonomic $\mathscr{D}_{Y}$-module, there exists a natural morphism

$(* *)$

$$
{ }^{p} \mathrm{DR}^{\mathrm{an}}\left(\varphi_{+} \mathcal{M}\right) \rightarrow \boldsymbol{R} \varphi_{*}{ }^{p} \mathrm{DR}^{\mathrm{an}}(\mathcal{M})
$$

where $\varphi_{*}$ denotes the direct image of sheaves and $\boldsymbol{R} \varphi_{*}$ denotes its right derived functor.

When $\varphi$ is proper, $(* *)$ is a quasi-isomorphism. When $\varphi$ is not proper and even if $\mathcal{M}$ is holonomic, it need not be so.

0.6. If $\varphi=\eta: U \hookrightarrow X$ as above, $(* *)$ is a quasi-isomorphism if $\eta_{+} M$ has regular singularities along $D$ (see [3, p. 326] or [17]). If $\mathcal{M}$ is only holonomic, the cone of $(* *)$ is defined to be the irregularity complex of $\eta_{+} \mathcal{M}$ along $D$ (see [18]). 
0.7. There is a duality functor for constructible complexes called Verdier duality, which gives usual Poincaré duality at the cohomology level. This functor $\boldsymbol{D}_{X}$ is compatible with the duality of holonomic $\mathscr{D}_{X}$-modules via ${ }^{p} \mathrm{DR}^{\mathrm{an}}$, namely $\boldsymbol{D}_{X}{ }^{p} \mathrm{DR}^{\text {an }} \boldsymbol{M}={ }^{p} \mathrm{DR}^{\text {an }} D_{X} \mathcal{M}$ (see [3, p. 326] or [16, p. 56]).

Consequently for $\varphi: Y \rightarrow X$ as above and under regularity assumptions on the holonomic $\mathscr{D}_{Y}$-module $\mathcal{M}$, the functor $\varphi_{\dagger}$ defined for holonomic modules corresponds via $\mathrm{DR}^{\text {an }}$ to the functor $\varphi_{\text {! }}$ of direct image with proper supports defined at the level of sheaves, namely $\varphi_{!}^{p} \mathrm{DR}^{\mathrm{an}} \mathcal{M}={ }^{p} \mathrm{DR}^{\mathrm{an}} \varphi_{\dagger} \mathcal{M}$.

0.8. Let $\mathscr{F}$ be a constructible complex on $X$. For each $x \in X$, put $\chi_{x}(\mathscr{F})=$ $\sum_{i}(-1)^{i} \operatorname{dim} \mathscr{H}^{i}(\mathscr{F})_{x}$, where $\mathscr{H}^{i}(\mathscr{F})$ is the $i$ th cohomology sheaf of $\mathscr{F}$, which is by assumption a constructible sheaf on $X$. Then $x \mapsto \chi_{x}(\mathscr{F})$ is a constructible function on $X$ with values in $\mathbf{Z}$ : it is constant on the strata of some algebraic stratification of $X$.

When $\mathscr{F}=\mathrm{DR}^{\text {an }} \mathcal{M}, \mathcal{M}$ a holonomic $\mathscr{D}_{X}$-module, this function allows one to recover the characteristic cycle of $\mathcal{M}$ (a union of Lagrangian varieties in the cotangent bundle $T^{*} X$ with multiplicities) (see for instance [6]).

The global Euler characteristics $\chi(X, \mathscr{F})=\sum_{i}(-1)^{i} \operatorname{dim} \boldsymbol{H}^{i}(X, \mathscr{F})$, where $\boldsymbol{H}^{\imath}$ denotes the $i$ th hypercohomology group of the complex $\mathscr{F}$, can also be recovered from the characteristic function of $\mathscr{F}$ and the usual Euler characteristics of the strata on which it is constant. For instance, if $\mathscr{F}$ is a local system on $X$, we have $\chi_{x}(\mathscr{F})=\operatorname{dim} \mathscr{F}_{x}=r$ for all $x$ and $\chi(X, \mathscr{F})=r \cdot \chi(X)$.

0.9. Let $h: X \rightarrow \mathbf{C}$ be a regular function on $X$ and let $\mathscr{F}$ be a constructible complex on $X$. The nearby cycle complex $\phi_{h} \mathscr{F}$ is a constructible complex on $h^{-1}(0)$ (see for instance $[10$, p. 350$]$ for the definition). It depends only on the restriction of $\mathscr{F}$ to $X-h^{-1}(0)$. If for instance this restriction is a local system of rank $r$, the characteristic function $x \mapsto \chi_{x}\left(\phi_{h} \mathscr{F}\right)\left(x \in h^{-1}(0)\right)$ is computed in the following way: let $F_{x}$ denote the Milnor fiber of $h$ at $x$; then $\chi_{x}\left(\phi_{h} \mathscr{F}\right)=$ $r \chi\left(F_{x}\right)$ where $\chi$ in the RHS is the usual Euler characteristics of $F_{x}$.

Coming back to the general situation one can also define (see for instance [10, p. 350]) the vanishing cycle complex $\phi_{h} \mathscr{F}$ as the cone of the natural morphism $i_{h^{-1}(0)}^{-1} \mathscr{F} \rightarrow \phi_{h} \mathscr{F}$ where $i_{h^{-1}(0)} h^{-1}(0) \hookrightarrow X$ denotes the inclusion.

\section{A global comparison theorem}

In order to formulate the result, denote $U^{\text {an }}$ the complex analytic manifold underlying $U$ and $\operatorname{DR}^{\text {an }}(\mathcal{N})$ the corresponding de Rham complex of $\mathcal{N}$. It is a 
bounded complex with constructible cohomology on $U^{\text {an }}$ (for instance it can be a local system on $U$ ). Let $\Phi$ be the family of closed sets of $U^{\text {an }}$ on which $e^{-f}$ is rapidly decreasing (a more precise definition will be given later).

\section{Theorem 1.1. One has $\boldsymbol{H}^{k}\left(U, \mathrm{DR}\left(\mathcal{M}_{f}\right)\right)=\boldsymbol{H}_{\Phi}^{k}\left(U^{\mathrm{an}}, \mathrm{DR}^{\mathrm{an}}(\mathcal{M})\right)$ for all $k$.}

This theorem is essentially proved in [8] (see also [15] and [22]). Indeed, as we shall see below, the RHS can be better understood: let $\rho>0$ and denote $H_{\rho}$ the half-space $\operatorname{Re}(-t) \geq \rho$ in $\mathbf{C}$; let $U_{\rho}=f^{-1}\left(H_{\rho}\right) \subset U^{\text {an }}$. Then we shall show that the RHS is equal to the relative hypercohomology $\boldsymbol{H}^{k}\left(U^{\mathrm{an}}, U_{\rho} ; \mathrm{DR}^{\mathrm{an}}(\mathcal{M})\right)$ for $\rho$ big enough. The restriction map

$$
\boldsymbol{H}^{k}\left(U^{\mathrm{an}}, U_{\rho} ; \mathrm{DR}^{\mathrm{an}}(\mathcal{M})\right) \rightarrow \boldsymbol{H}^{k}\left(U^{\mathrm{an}}, f^{-1}(t) ; \mathrm{DR}^{\mathrm{an}}(\mathcal{M})\right)
$$

induces then an isomorphism for $t \in H_{\rho}$ and so the LHS is also isomorphic to the relative hypercohomology group $\boldsymbol{H}^{k}\left(U^{\text {an }}, f^{-1}(t) ; \mathrm{DR}^{\text {an }}(M)\right)$ for a general fiber $f^{-1}(t)$. With this formulation, the result is proved in [8], [15] and [22] when $U=$ $\mathbf{C}^{l}$, but the proof extends easily to the general case. We shall give below another proof (in the style of [15]) which can be adapted to prove also the local comparison Theorem 5.1.

In particular we obtain an equality of Euler characteristics (see $\S 0.8$ ):

Corollary 1.2. Assume $\mathcal{M}$ is smooth of rank $r$ on $U$ and that $U$ is affine. Then

$$
\chi\left(\operatorname{DR}\left(M_{f}(U)\right)\right)=r\left[\chi\left(U^{\mathrm{an}}\right)-\chi\left(f^{-1}(t)\right)\right]
$$

where $t$ is a generic value for $f$.

Proof of Theorem 1.1. We shall sheafify the formulation of the theorem. This will be useful for the local comparison Theorem 5.1. In fact we shall reduce the global comparison theorem to a local one in dimension 1 .

Let $\pi: \tilde{\mathbf{P}}^{1} \rightarrow \mathbf{P}^{1}$ be the oriented real blow-up of $\mathbf{P}^{1}$ at infinity. This is the space of polar coordinates at infinity, which is diffeomorphic to the disc, and $\pi^{-1}(\infty)=S^{1}$ is the circle of directions at $\infty$. We shall write $\tilde{\mathbf{P}}^{1}=\mathbf{C} \cup S^{1}$. One has $\tilde{\mathbf{P}}^{1}-\{0\} \simeq \mathbf{R}_{+} \times S^{1}$ and $\pi$ is given by $1 / t=\rho e^{i \theta}$.

Let $X$ be a compactification of $U$ such that $f$ extends as a projective map $F$ : $X \rightarrow \mathbf{P}^{1}$. One can extend $F$ to $\tilde{F}: \tilde{X} \rightarrow \tilde{\mathbf{P}}^{1}$, where $\tilde{X}$ is the fiber product $X \times{ }_{\mathbf{P}^{1}} \tilde{\mathbf{P}}^{1}$. It is a real semi-algebraic space and $\tilde{F}$ is a semi-algebraic map.

Let $I$ be the open interval in $S^{1}$ defined as the set of directions in the neighbourhood of which $e^{-t}$ is decreasing, i.e. $\left.\theta \in\right]-\pi / 2, \pi / 2\left[\right.$. Denote $\tilde{X}_{I}$ the in- 
verse image of $\mathbf{C} \cup I$ in $\tilde{X}$. The family $\Phi$ of closed sets considered in the theorem is defined as follows: a closed set $A$ of $U$ is in $\Phi$ if its closure in $\tilde{X}$ is contained in $\tilde{X}_{I}$.

In order to sheafify the previous construction, consider the following inclusions

$$
U \stackrel{\alpha}{\hookrightarrow} \tilde{X}_{I} \stackrel{\beta}{\hookrightarrow} \tilde{X}
$$

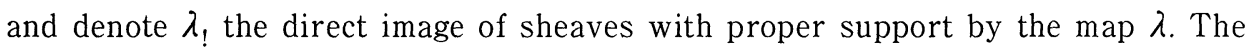
hypercohomology with supports in $\Phi$ considered in the theorem is the hypercohomology on $\tilde{X}$ of the complex of sheaves $\beta, \boldsymbol{R} \alpha_{*} \mathrm{DR}^{\text {an }}(\mathcal{M})$.

Remark. In contrast, the comparison theorem for regular holonomic modules applied to $\mathcal{M}$ implies that $\mathbf{R} \Gamma(U, \mathrm{DR}(\mathcal{M}))$ is the hypercohomology on $\tilde{X}$ of the complex $\mathbf{R} \beta_{*} \mathbf{R} \alpha_{*} \mathrm{DR}^{\mathrm{an}}(\mathcal{M})$.

Now, the hypercohomology $\boldsymbol{H}^{k}\left(\tilde{X}, \beta_{!} \boldsymbol{R} \alpha_{*} \mathrm{DR}^{\text {an }}(\mathcal{M})\right)$ is also equal to the relative hypercohomology $\boldsymbol{H}^{k}\left(\tilde{X}, \tilde{X}-\tilde{X}_{I} ; \boldsymbol{R} \beta_{*} \mathbf{R} \alpha_{*} \mathrm{DR}^{\text {an }}(\mathcal{M})\right)$, and this one is isomorphic to $\boldsymbol{H}^{k}\left(U, U_{\rho} ; \mathrm{DR}^{\text {an }}(\mathcal{M})\right)$ for $\rho$ large enough.

By direct image by $f$ we shall now reduce to the case where $U=\mathbf{C}$ and $f=$ Id (but $\mathcal{M}$ is replaced with a bounded complex with regular holonomic cohomology on $\mathbf{C}$ ). First, let $f_{+}$be the direct image functor for $\mathscr{D}_{U}$-modules (see $\S 0.2$ ). Then one has

$$
f_{+}\left(M_{f}\right)=\left(f_{+} M\right)_{\mathrm{Id}}=\left(f_{+} M\right) \otimes e^{t} .
$$

Moreover, using the relative comparison theorem for regular holonomic modules (because $f$ is not proper) we have (see $\S 0.5$ )

$$
{ }^{p} \mathrm{DR}^{\mathrm{an}}\left(f_{+} \mathcal{M}\right)=\boldsymbol{R} f_{*}^{p} \mathrm{DR}^{\mathrm{an}}(\mathcal{M}) .
$$

If we continue to denote $\alpha$ and $\beta$ the inclusions $\mathbf{C} \hookrightarrow \mathbf{C} \cup I$ and $\mathbf{C} \cup I \hookrightarrow \tilde{\mathbf{P}}^{1}$ we are reduced to showing that

$$
\operatorname{DR}\left(\mathcal{N}_{\text {Id }}^{\cdot}(\mathbf{C})\right)=\boldsymbol{R} \Gamma\left(\tilde{\mathbf{P}}^{1}, \beta_{!} \boldsymbol{R} \alpha_{*} \operatorname{DR}^{\text {an }}\left(\mathcal{N}^{*}\right)\right)
$$

for any bounded complex $\mathcal{N}^{\bullet}$ on $\mathbf{C}$ with regular holonomic cohomology (we shall apply this to $f_{+} M$ ).

We shall prove this equality at the sheaf level. In order to do this, we must realize the LHS as the hypercohomology of a complex of sheaves on $\tilde{\mathbf{P}}^{1}$. Let $j: \mathbf{C}$ $\hookrightarrow \mathbf{P}^{1}$ denote the inclusion and $j_{+}$be the corresponding direct image of $\mathscr{D}_{\mathrm{c}}$-modules. We then have 


$$
\begin{aligned}
\operatorname{DR}\left(\mathcal{N}_{\mathrm{Id}}^{\cdot}(\mathbf{C})\right) & =\boldsymbol{R} \Gamma\left(\mathbf{P}^{1}, \mathrm{DR}\left(j_{+} \mathcal{N}_{\mathrm{Id}}^{\cdot}\right)\right) \text { by definition } \\
& =\boldsymbol{R} \Gamma\left(\mathbf{P}^{1}, \mathrm{DR}^{\mathrm{an}}\left(j_{+} \mathcal{N}_{\mathrm{Id}}^{\cdot}\right)\right) \text { by GAGA }
\end{aligned}
$$

Following Malgrange (see [14, Chap. IV § 4]) we shall construct on $\tilde{\mathbf{P}}^{1}$ a complex denoted $\widetilde{\mathrm{DR}}^{\text {mod }}\left(j_{+} \mathcal{N}_{\text {Id }}^{\cdot}\right)$ such that

$$
\mathrm{DR}^{\mathrm{an}}\left(j_{+} \mathcal{N}_{\mathrm{Id}}^{\cdot}\right)=\boldsymbol{R} \pi_{*} \widetilde{\mathrm{DR}}^{\mathrm{mod}}\left(j_{+} \mathcal{N}_{\mathrm{ld}}^{\cdot}\right) .
$$

We shall then compare the two complexes $\widetilde{\mathrm{DR}}^{\text {mod }}\left(j_{+} \mathcal{N}_{\mathrm{Id}}^{\cdot}\right)$ and $\beta_{!} \boldsymbol{R} \alpha_{*}$. $\operatorname{DR}^{\text {an }}\left(\mathcal{N}^{*}\right)$ which both live on $\tilde{\mathbf{P}}^{1}$.

Let $\mathscr{A}^{\text {mod }} \subset \beta_{*} \alpha_{*} \mathscr{O}_{\mathbf{C}}^{\text {an }}$ be the subsheaf of sections with moderate growth along $S^{1}$. It is equal to $\mathscr{O}_{\mathbf{C}}^{\text {an }}$ outside $S^{1}$. One has

LEMma $1.3 . \quad \pi_{*} \mathscr{A}^{\mathrm{mod}}=\mathscr{O}_{\mathbf{P}^{1}}^{\mathrm{an}}[*(\infty)]$ and $\boldsymbol{R}^{i} \pi_{*} \mathscr{A}^{\mathrm{mod}}=0$ for $i \neq 0$

Sketch of proof. The sheaf $\mathscr{A}^{\text {mod }}$ admits a resolution by currents with moderate growth along $S^{1}$ (the dual of the sheaf of $\mathscr{C}^{\infty}$ functions which are flat along $S^{1}$ ) and by direct image by $\pi$ one gets the Dolbeaut-Grothendieck complex (of currents with moderate growth at the origin) on $\mathbf{C}$ which is a resolution of $\mathscr{O}_{\mathbf{P}^{1}}^{\text {an }}[*(\infty)]$.

It is easy to verify that $\mathscr{A}^{\text {mod }}$ is flat over $\pi^{-1} \mathscr{O}_{\mathbf{P}^{1}}^{\text {an }}$ so if we put

$$
\widetilde{\mathrm{DR}}^{\text {mod }}\left(j_{+} \mathcal{N}_{\text {Id }}^{\cdot}\right) \stackrel{\text { def }}{=} \mathscr{A}^{\text {mod }} \bigotimes_{\pi^{-1} \mathscr{P}_{\mathbb{P}^{2}}^{\text {an }}} \mathrm{DR}^{\text {an }}\left(j_{+} \mathcal{N}_{\text {Id }}^{\cdot}\right)
$$

we have, due to the projection formula

$$
\mathrm{DR}^{\mathrm{an}}\left(j_{+} \mathcal{N}_{\mathrm{Id}}^{\cdot}\right)=\boldsymbol{R} \pi_{*} \overline{\mathrm{DR}}^{\mathrm{mod}}\left(j_{+} \mathcal{N}_{\mathrm{Id}}^{\cdot}\right) .
$$

We want to show that there exists a quasi-isomorphism

$$
\widetilde{\mathrm{DR}}^{\mathrm{mod}}\left(j_{+} \mathcal{N}_{\mathrm{Id}}^{\cdot}\right) \simeq \beta ! R \alpha_{*} \mathrm{DR}^{\mathrm{an}}\left(\mathcal{N}^{*}\right) .
$$

Remark first that we have a natural morphism

$$
\widetilde{\mathrm{DR}}^{\mathrm{mod}}\left(j_{+} \mathcal{N}_{\mathrm{Id}}^{\cdot}\right) \rightarrow \boldsymbol{R} \beta_{*} \boldsymbol{R} \alpha_{*} \mathrm{DR}^{\mathrm{an}}\left(\mathcal{N}^{*}\right) .
$$

In order to see that it factorizes through $\beta_{1} \boldsymbol{R} \alpha_{*} \mathrm{DR}^{\mathrm{an}}\left(\mathcal{N}^{*}\right)$, it is enough to prove that if $\gamma: S^{1}-I \hookrightarrow \tilde{\mathbf{P}}^{1}$ denotes the closed inclusion, the restriction $\gamma^{-1}$. $\mathrm{DR}^{\text {mod }}\left(j_{+} \mathcal{N}_{\text {Id }}^{*}\right)$ is zero. This statement can be reduced by a simple extension argument to the case where $j_{+} \mathcal{N}^{\cdot}$ is a single meromorphic connection near $\infty \in \mathbf{P}^{1}$ and we can assume that it is of rank one as an $\mathscr{O}_{\mathbf{P}^{1}}^{\text {an }}[*(\infty)]$-module.

Once the existence of the morphism is proved, the fact that it is a quasi- 
isomorphism can also be reduced to the case of rank one meromorphic connections near $\infty$. Moreover both results can be proved locally on $\tilde{\mathbf{P}}^{1}$.

We shall then assume that $j_{+} \mathcal{N}$ is the meromorphic connection near $\infty$ generated by $t^{a}$ for some $a \in \mathbf{C}^{*}$ and near $\infty$ the complex $\widetilde{\mathrm{DR}}^{\text {mod }}\left(j_{+} \mathcal{N}_{\text {Id }}\right)$ is the complex

$$
0 \rightarrow \mathscr{A}^{\mathrm{mod}} \stackrel{t^{-a} e^{-t} \partial_{t} t^{a} e^{t}}{\longrightarrow} \mathscr{A}^{\text {mod }} \rightarrow 0 .
$$

Locally on $\tilde{\mathbf{P}}^{1}, t^{a}$ and $t^{-a}$ define local sections of $\mathscr{A}^{\text {mod }}$ so this complex is locally isomorphic to

$$
0 \rightarrow \mathscr{A}^{\mathrm{mod}} \stackrel{e^{-t} \partial_{t} e^{t}}{\longrightarrow} \mathscr{A}^{\text {mod }} \rightarrow 0
$$

and there remains to show that this complex is 0 when restricted to $S^{1}-I$ and is equal to the constant sheaf (in degree 0 ) on $I$. These properties are clearly satisfied by $\operatorname{Ker} e^{-t} \partial_{t} e^{t}: \mathscr{A}^{\text {mod }} \rightarrow \mathscr{A}^{\text {mod }}$ so the proof is now reduced to the following

Lemma 1.5. The map $e^{-t} \partial_{t} e^{t}: \mathscr{A}^{\mathrm{mod}} \rightarrow \mathscr{A}^{\mathrm{mod}}$ is onto.

The proof of this lemma can be done exactly as in [14, Appendice 1 p. 211] and is analogous to Lemma 3.8, Chap. IV in loc. cit.

\section{The case of isolated singularities}

Consider now the following general situation: let $S$ be a complex analytic space of pure dimension $l$ equipped with a complex analytic Whitney stratification \&. Let $g: S \rightarrow \mathbf{C}$ be a holomorphic function. One says (see [12]) that $g$ has only isolated singularities on $(S, \mathscr{S})$ if the restriction of $g$ to each stratum $S_{\alpha}$ has only isolated critical points (in the usual sense, since $S_{\alpha}$ is smooth). Such points are the critical points of $g$ with respect to $\mathscr{S}$.

Proposition 2.1. Let $g$ has isolated singularities on $(S, \&)$ and let $\mathscr{F}$ be any perverse complex on $S$ which is constructible with respect to $\&$. Let $c \in \mathbf{C}$ and let $\phi_{g-c} \mathscr{F}$ be the complex of vanishing cycles of $\mathscr{F}$ along the fiber $g^{-1}(c)$ (see $\left.§ 0.9\right)$. Then the perverse complex $\phi_{g-c} \mathscr{F}$ is supported on the critical points of $g$ on the fiber $g^{-1}(c)$ and the cohomology of this complex is nonzero only in degree -1 at most.

Proof. Let $x \in S_{\alpha}$ and assume that $x$ is not a critical point of $g_{\mid S_{\alpha}}$. Then locally around $x$ the map $g:(S, \&) \rightarrow \mathbf{C}$ is homeomorphic to the projection of the product $\left(g^{-1}(g(x)), \mathscr{\bigotimes}_{\mid g^{-1}(g(x))}\right) \times \mathbf{C}$ to $\mathbf{C}$. This proves that any complex $\mathscr{F}$ constructi- 
ble with respect to $\mathscr{F}$ has no vanishing cycles at $x$. For any such $\mathscr{F}$ and any $c \in \mathbf{C}$ the complex of vanishing cycles $\phi_{g-c} \mathscr{F}$ is then supported on the isolated critical points of $g$ on $g^{-1}(c)$. Now, if moreover $\mathscr{F}$ is perverse on $S$, the complex $\phi_{g-c} \mathscr{F}$ shifted by -1 is perverse on $g^{-1}(c)$ (see [7] or [21]) and is supported on isolated points. This implies that the cohomology of $\phi_{g-c} \mathscr{F}$ is nonzero in degree -1 only.

Let us now come back to the original situation. Let $X$ be a compactification of $U$ (of dimension $l$ ) on which $f$ extends as a mapping $F: X \rightarrow \mathbf{P}^{1}$ and let $Y=X-$ $F^{-1}(\infty)$.

Proposition 2.2. Assume that $U$ is affine, $Y$ is smooth, $Y-U$ is a divisor and that there exists a complex analytic Whitney stratification $Y$ of $Y$ such that

1. $U^{\mathrm{an}}$ is a union of strata and $\mathrm{DR}^{\mathrm{an}}(\mathcal{M})$ is constructible with respect to $\mathscr{Y}_{\mid U^{\mathrm{an}}}$;

2. $F$ has isolated singularities on $(Y, \mathscr{Y})$.

Then $\mathrm{DR}\left(M_{f}(U)\right)$ has nonzero cohomology in degree l at most.

Proof. Remark first that if $t$ is sufficiently general, we have

$$
i_{t}^{-1} \boldsymbol{R} f_{*} \mathrm{DR}^{\mathrm{an}}(\mathcal{M})=\boldsymbol{R} f_{*} i_{f^{-1}(t)}^{-1} \mathrm{DR}^{\mathrm{an}}(\mathcal{M})
$$

where $i_{t}$ and $i_{f^{-1}(t)}$ are the inclusions $\{t\} \hookrightarrow \mathbf{C}$ and $f^{-1}(t) \hookrightarrow U^{\text {an }}$. Consequently, for $t$ general enough, the relative cohomology $\boldsymbol{H}^{k}\left(U^{\mathrm{an}}, f^{-1}(t) ; \mathrm{DR}^{\mathrm{an}}(\mathcal{M})\right)$ is equal to the relative cohomology $\boldsymbol{H}^{k}\left(\mathbf{C}, t ; R f_{*} \mathrm{DR}^{\text {an }}(\mathcal{M})\right)$ and is also equal, by Theorem 1.1 to $H^{k}\left(\mathrm{DR}\left(\mathcal{M}_{f}(U)\right)\right)$ since $U$ is affine.

Consider the perverse complex $\mathscr{P} \stackrel{\text { def }}{=}{ }^{p} \mathrm{DR}^{\mathrm{an}}(\mathcal{M})=\mathrm{DR}^{\mathrm{an}}(\mathcal{M})[l]$. We want to show that $\boldsymbol{H}^{k}\left(U^{\text {an }}, f^{-1}(t) ; \mathscr{P}\right)=0$ for $k \neq 0$. Consider the perverse cohomology sheaves ${ }^{p} \boldsymbol{R}^{m} f_{*} \mathscr{P}$ on $\mathbf{C}$. Since $f$ is affine we have ${ }^{p} \boldsymbol{R}^{m} f_{*} \mathscr{P}=0$ for $m>0$ (see $[10$, Theorem 10.3.17]). We shall prove that ${ }^{p} \boldsymbol{R}^{m} f_{*} \mathscr{P}$ are local systems for $m<0$. Let $\eta: U \hookrightarrow Y$ be the inclusion. Then $\boldsymbol{R} \eta_{*} \mathscr{P}$ is perverse on $Y$ and is constructible with respect to $\mathscr{Y}$. Let $c \in \mathbf{C}$ and consider the vanishing cycle functor $\phi_{F-c}$ on $Y$ and $\phi_{\tau-c}$ on $\mathbf{C}$, where $\tau$ denotes the identity function on $\mathbf{C}$. Put ${ }^{\mathrm{p}} \phi=\phi[-1]$. Then these functors commute with the proper direct image $\boldsymbol{R} F_{*}$ and also with the perverse cohomology ([7]). Hence we have

$$
\begin{aligned}
{ }^{\mathrm{p}} \phi_{\tau-c}{ }^{\mathrm{p}} \boldsymbol{R}^{\mathrm{m}} f_{*} \mathscr{P} & ={ }^{p} \phi_{\tau-c}{ }^{p} \boldsymbol{R}^{m} F_{*}\left(\boldsymbol{R} \eta_{*} \mathscr{P}\right) \\
& ={ }^{p} \boldsymbol{R}^{m} F_{*}\left({ }^{p} \phi_{F-c} \boldsymbol{R} \eta_{*} \mathscr{P}\right) .
\end{aligned}
$$

By the previous proposition ${ }^{p} \phi_{F-c} \boldsymbol{R} \eta_{*} \mathscr{P}$ is a perverse sheaf supported on points and its direct image has perverse cohomology in degree 0 only. Thus ${ }^{\mathrm{p}} \boldsymbol{R}^{m} f_{*} \mathscr{P}$ has 
no vanishing cycle at any $c \in \mathbf{C}$ for $m<0$, so is a local system on $\mathbf{C}$, i.e. a constant sheaf, up to a shift by 1 .

Because a constant sheaf has no nonzero relative hypercohomology, we see by an easy induction that

$$
\boldsymbol{H}^{k}\left(U, f^{-1}(t) ; \mathscr{P}\right)=H^{k}(C,\{t\} ; \mathscr{F})
$$

where $\mathscr{F}$ is the perverse sheaf ${ }^{p} \boldsymbol{R}^{0} f_{*} \mathscr{P}$. The result follows now from

Lemma 2.3. Let $\mathscr{F}$ be any perverse sheaf on $\mathbf{C}$. Then for $t$ general enough (i.e. not in the singular set $\sum$ of $\left.\mathscr{F}\right)$, we have $\boldsymbol{H}^{k}(\mathbf{C},\{t\} ; \mathscr{F})=0$ for $k \neq 0$.

Proof. We know that $\boldsymbol{H}^{k}(\mathbf{C} ; \mathscr{F})=0$ for $k>0$ and $k \leq-2$ because $\mathbf{C}$ is affine (see e.g. [10, Theorem 10.3.8]). Moreover we have $\boldsymbol{H}^{k}(\{t\} ; \mathscr{F})=0$ for $k \neq$ -1 if $t \notin \sum$. Hence we have $\boldsymbol{H}^{k}(\mathbf{C},\{t\} ; \mathscr{F})=0$ for $k \neq 0,-1$.

Assume that $\sum$ is nonempty (otherwise the result is clear) and let $\kappa: \mathbf{C}-\Sigma$ $\hookrightarrow \mathbf{C}$ be the inclusion. Then $\kappa^{-1} \mathscr{F}=\mathscr{L}[1]$ where $\mathscr{L}$ is a local system on $\mathbf{C}-\Sigma$, and $\boldsymbol{H}^{-1}\left(\mathbf{C} ; \kappa, \kappa^{-1} \mathscr{F}\right)=0$, i.e. $H^{0}\left(\mathbf{C}, \kappa_{!} \mathscr{L}\right)=0$, because $\Sigma$ is nonempty.

Let us now prove that $\boldsymbol{H}^{-1}(\mathbf{C},\{t\} ; \mathscr{F})=0$. Let $\sigma \in \boldsymbol{H}^{-1}(\mathbf{C} ; \mathscr{F})$ be such that its image in $\mathscr{H}^{-1}\left(i_{t}^{-1} \mathscr{F}\right)$ is zero. Let $\mathscr{V}$ be a small neighbourhood of $c \in \sum$. We may assume that $t \in \mathscr{V}$. Then the map $\boldsymbol{H}^{-1}(\mathscr{V} ; \mathscr{F}) \rightarrow \mathscr{H}^{-1}\left(i_{t}^{-1} \mathscr{F}\right)$ is equal to the map $\mathscr{H}^{-1}\left(i_{c}^{-1} \mathscr{F}\right) \rightarrow{ }^{p} \phi_{\tau-c} \mathscr{F}$, with ${ }^{p} \phi=\phi[-1]$. But $\mathscr{F}$ being perverse, we have an exact sequence of vector spaces

$$
0 \rightarrow \mathscr{H}^{-1}\left(i_{t}^{-1} \mathscr{F}\right) \rightarrow^{p} \phi_{\tau-c} \mathscr{F} \rightarrow{ }^{p} \phi_{\tau-c} \mathscr{F} \rightarrow \mathscr{H}^{0}\left(i_{t}^{-1} \mathscr{F}\right) \rightarrow 0 .
$$

We deduce from this that the image of $\sigma$ in $\boldsymbol{H}^{-1}(\mathscr{V} ; \mathscr{F})$ is zero, and applying this to all $c \in \sum$ we obtain that $\sigma$ is in the image of $\boldsymbol{H}^{-1}\left(\mathbf{C} ; \kappa, \kappa^{-1} \mathscr{F}\right) \rightarrow \boldsymbol{H}^{-1}(\mathbf{C} ; \mathscr{F})$, which is zero by the previous argument.

\section{The case of generic monodromy}

Let $Z$ be a smooth partial compactification of $U$ and let $j: U \hookrightarrow Z$ be the open inclusion. A holonomic $\mathscr{D}_{U}$-module admits many extensions as a holonomic $\mathscr{D}_{Z}$-module. One is denoted $j_{+} \mathcal{N}$, another is $j_{\dagger} \mathcal{N} \stackrel{\text { def }}{=} \mathscr{D}_{X} j_{+} D_{U} \mathcal{N}$ (see $\S 0.4$ ) where $\mathscr{D}_{X}$ (resp. $\mathscr{D}_{U}$ ) is the duality functor for holonomic $\mathscr{D}_{X}$ or $\mathscr{D}_{U}$-modules (see $\S 0.3$ ). One has a natural morphism:

$$
j_{+} \mathcal{N} \rightarrow j_{+} \mathcal{N}
$$


Proposition 3.1. Assume that $U$ is affine of dimension $l$ and let $M$ be a holonomic $\mathscr{D}_{U}$-module. Assume that there exists a smooth compactification $X$ of $U$ satisfying the following properties:

1. $f$ extends to $F: X \rightarrow \mathbf{P}^{1}$;

2. let $Y=X-F^{-1}(\infty)$ and $j: U \hookrightarrow Y$ be the inclusion; then the natural morphism $j_{+} \mathcal{M} \rightarrow j_{+} \mathcal{M}$ is an isomorphism.

Then $\operatorname{DR}\left(M_{f}(U)\right)$ has cohomology in degree l at most.

Proof. Let $\eta: U \hookrightarrow X$ be the inclusion. It is enough to prove that the morphism $\eta_{+} M_{f} \rightarrow \eta_{+} \mathcal{M}_{f}$ is an isomorphism, or equivalently that the dual $D_{X}\left(\eta_{+} M\right)$ is equal to $\eta_{+}\left(D_{U} \mathcal{M}_{f}\right)$ (it is a priori equal to $\eta_{\dagger}\left(D_{U} \mathcal{M}_{f}\right)$ ). Indeed, if this is the case, and because $U$ is affine, one has

$$
\begin{aligned}
\boldsymbol{R} \Gamma\left(X^{\mathrm{an}},{ }^{p} \mathrm{DR}^{\mathrm{an}}\left(\eta_{+} \mathcal{M}_{f}\right)\right) & =\boldsymbol{R} \Gamma\left(X,{ }^{p} \mathrm{DR}\left(\eta_{+} \mathcal{M}_{f}\right)\right)(\text { by GAGA }) \\
& ={ }^{p} \mathrm{DR}\left(\mathcal{M}_{f}(U)\right)
\end{aligned}
$$

so this complex has nonzero cohomology in degree $\leq 0$ at most. The same is true (for the same reasons) for the complex

$$
\begin{aligned}
{ }^{p} \operatorname{DR}\left(D_{U} \mathcal{M}_{f}(U)\right) & =\boldsymbol{R} \Gamma\left(X,{ }^{p} \mathrm{DR}\left(\eta_{+} D_{U} M_{f}\right)\right) \\
& =\boldsymbol{R} \Gamma\left(X,{ }^{p} \mathrm{DR}\left(D_{X} \eta_{+} \mathcal{M}_{f}\right)\right) \text { (by assumption) } \\
& =\boldsymbol{R} \Gamma\left(X,{ }^{p} \mathrm{DR}^{\mathrm{an}}\left(D_{X} \eta_{+} \mathcal{M}_{f}\right)\right) \text { (by GAGA) } \\
& =\boldsymbol{R} \Gamma\left(X, \boldsymbol{D}^{p} \mathrm{DR}^{\mathrm{an}}\left(\eta_{+} \mathcal{M}_{f}\right)\right) \text { by the local duality theorem } \\
& =\boldsymbol{D} \boldsymbol{R} \Gamma\left(X,{ }^{p} \mathrm{DR}^{\mathrm{an}}\left(\eta_{+} \mathcal{M}_{f}\right)\right) \text { by Poincaré-Verdier duality }
\end{aligned}
$$

where $\boldsymbol{D}$ is the Verdier duality for constructible complexes. We conclude that $\boldsymbol{R} \Gamma\left(X,{ }^{p} \mathrm{DR}^{\mathrm{an}}\left(\eta_{+} M_{f}\right)\right)$ has nonzero cohomology in degree $\geq 0$ at most, and putting all together, $\boldsymbol{R} \Gamma\left(X, \mathrm{DR}^{\mathrm{an}}\left(\eta_{+} \mathcal{M}_{f}\right)\right)$ has nonzero cohomology in degree $l$ at most.

Let us now prove that the dual $D_{X} \eta_{+} M_{f}$ is equal to $\eta_{+} D_{U} M_{f}$. Remark first that for a holonomic $\mathscr{D}_{U}$-module, we have $D_{U}\left(M_{f}\right)=\left(D_{U} M\right)_{-f}$ : indeed, choose a resolution of $\mathcal{M}$ by left $\mathscr{D}_{U}$-modules isomorphic to $\mathscr{D}_{U}^{p}$; we get a resolution of $\mathcal{M}_{f}$ by twisting the left structure of each term by $e^{f}$ and the resulting modules remain free; hence $\mathscr{E} x t_{\mathscr{D}_{U}}^{l}\left(\mathcal{M}_{f}, \mathscr{D}_{U}\right)=\mathscr{E} x t_{\mathscr{D}_{U}}^{l}\left(\mathcal{M}, \mathscr{D}_{U}\right) \otimes e^{f}$ as a right $\mathscr{D}_{U}$-module, and going from right to left by adjunction, we get the result. Let $\mathcal{M}^{\prime}=j_{+} \mathcal{M}=j_{+} \mathcal{M}$, which is a regular holonomic $\mathscr{D}_{Y^{-}}$module. We have

$$
\begin{aligned}
\mathcal{M}_{F}^{\prime} & =\left(j_{+} \mathcal{M}\right)_{F}=j_{+}\left(\mathcal{M}_{f}\right) \\
& =\left(j_{\dagger} \mathcal{M}\right)_{F}=j_{\dagger}\left(\mathcal{M}_{f}\right) \text { (previous remark). }
\end{aligned}
$$


Let $\eta^{\prime}: Y \hookrightarrow X$ be the open inclusion. Because of hypothesis (2), it is enough to prove that $D_{X} \eta_{+}^{\prime} \mathcal{M}_{F}^{\prime}=\eta_{+}^{\prime} D_{Y} \mathcal{M}_{F}^{\prime}$ because $\eta_{+}^{\prime} \mathcal{M}_{F}^{\prime}=\eta_{+} \mathcal{M}_{f}$ and $D_{Y} \mathcal{M}_{F}^{\prime}=D_{Y} j_{+} M_{f}=$ $j_{+} D_{U} \mathcal{M}_{f}$. Moreover, we may assume that $\mathcal{M}_{F}^{\prime}$ is a single holonomic $\mathscr{D}_{Y}$-module since the functors $\eta_{+}^{\prime}, D_{X}$ and $D_{Y}$ preserve perversity. From the characteristic property of $\eta_{+}^{\prime}$ (see $\S 0.4$ ) we have a natural morphism

$$
D_{X} \eta_{+}^{\prime} \mathcal{M}_{F}^{\prime} \rightarrow \eta_{+}^{\prime} D_{Y} \mathcal{M}_{F}^{\prime}
$$

the kernel and cokernel of which are supported on $F^{-1}(\infty)$.

Lemma 3.3. Every submodule of $\eta_{+}^{\prime} \mathcal{M}_{F}^{\prime}$ is of the form $\eta_{+}^{\prime} \mathcal{N}_{F}$ where $\mathcal{N}$ is a $\mathscr{D}_{Y^{-}}$submodule of $\mathcal{M}^{\prime}$.

Proof. This lemma is a direct consequence of [4, Prop. 1]. First, remark that if $\mathcal{N}$ exists, it is unique, because it is equal to the restriction of the submodule to $Y$ twisted by $e^{-F}$. Thus the assertion is local on $X$. Let $h=1 / F$. It is proved in loc. cit. that for each local section $m$ of $\eta_{+}^{\prime} \mathcal{M}^{\prime}$ there exists a functional equation

$$
m e^{1 / h} h^{s}=P m e^{1 / h} h^{s+1}
$$

where $P$ is a local section of $\mathscr{D}_{X}[s]$ (in this local situation we use analytic differential operators). The submodule that we consider admits a local generator of the form $m e^{1 / h}$ because it is holonomic, i.e. it is equal to the submodule $\mathscr{D}_{X} \cdot m e^{1 / h}$ of $\eta_{+}^{\prime} \mathcal{M}_{F}^{\prime}$. Specializing the functional equation to $s=-k, k \in \mathrm{N}$, one shows that, for every such $k,(1 / h)^{k} m$ is contained in this module, hence it is also equal to $\left(\mathscr{D}_{X} \cdot m\right)[1 / h] e^{1 / h}$.

From this lemma we also deduce that $\eta_{+}^{\prime} \mathcal{M}_{F}^{\prime}$ cannot have a nonzero quotient supported by $F^{-1}(\infty)$ (such a quotient is also of the form $\eta_{+}^{\prime} \mathcal{N}_{F}^{\prime}$ where $\mathcal{N}^{\prime}$ is some quotient of $\mathcal{M}^{\prime}$ ). By taking duals, we see that the kernel of (3.2) is 0 . Because we have $D_{Y} \mathcal{M}_{F}^{\prime}=\left(D_{Y} \mathcal{M}^{\prime}\right)_{-F}$, we conclude that (3.2) is onto by the same argument.

Remark. The assumption in 3.1 that $X$ is smooth was made only to simplify the argument. In fact, if $X$ is a projective compactification of $U$ and $i: X \hookrightarrow \mathbf{P}^{N}$ is some embedding, then one can define the functors $(i \circ j)_{\dagger}$ and $(i \circ j)_{+}$. The condition that $(i \circ j)_{+} \mathcal{M} \rightarrow(i \circ j)_{+} \mathcal{M}$ is an isomorphism is independent of the choice of the embedding and, because $\mathcal{M}$ is regular, is equivalent to the fact that $j_{!} \mathrm{DR}^{\text {an }}(\mathcal{M}) \rightarrow \boldsymbol{R} j_{*} \mathrm{DR}^{\mathrm{an}}(\mathcal{M})$ is a quasi-isomorphism. 


\section{Applications to arrangements of hyperplanes}

We shall use the notations of [1]: let $V$ be a complex affine space of dimension $l, \mathscr{G}=\left\{A_{1}, \ldots, A_{p}\right\}$ be an arrangement of hyperplane. We make no assumption of general position. Let $N(\mathscr{G})$ be the union of hyperplanes in $\mathscr{G}$ and $U=M(\mathscr{G})=V$ $-N(\mathscr{G})$. Let $f$ be a polynomial on $V$. It induces a regular function $f$ on the affine open set $U$. Let $\mu=\left\{\mu_{1}, \ldots, \mu_{p}\right\}$ be a set of nonzero complex numbers and let $\mathscr{L}_{\mu}$ be the local system on $U$ with monodromy $\mu_{i}$ around $A_{i}$. Then $\mathcal{M}=\mathscr{O}_{U} \otimes_{\mathbf{C}} \mathscr{L}_{\mu}$ is a regular holonomic $\mathscr{D}_{U}$-module and its analytic de Rham complex reduces to the local system $\mathscr{L}_{\mu}$. The algebraic de Rham complex $\mathrm{DR}\left(\mathscr{M}_{f}(U)\right)$ is exactly the complex denoted $\left(\Omega^{*}(* \mathscr{G}), \nabla_{f}\right)$ in $[1]$.

The assumption of general position for $\mathscr{G}$ is only needed for proving the equality between this complex and the corresponding logarithmic one. Because the latter will not be considered here, we shall not make this assumption in what follows.

A conjecture of [1]. Now, Theorem 1.1 is exactly the conjecture in [1], for all cohomology groups, without any assumption of general position for $\mathscr{G}$ or of $\overline{\mathscr{G}}_{-}$ transversality for $f$.

The case where $f$ is $\bar{G}$-transverse. We shall now prove as a consequence of Proposition 2.2 an analogue of [1, Theorem 10.3-(3)].

Proposition 4.1. Assume that $f$ is $\bar{G}$-transverse. Then $\operatorname{DR}\left(M_{f}(U)\right)$ has cohomology in degree $l$ at most.

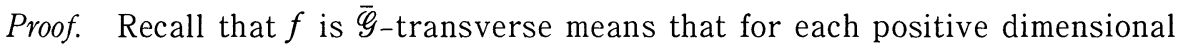
facet of the arrangement $\mathscr{G}$ (including $V$ itself), the restriction of the homogeneous part of maximal degree $f_{d}$ of $f=\sum_{k=0}^{d} f_{k}$ to the direction of this facet has no critical point outside the origin. Consider now the subset $G \subset \mathbf{P}^{l} \times \mathbf{C}$ defined by the equation

$$
f_{d}(x)+z f_{d-1}(x)+\cdots+z^{d} f_{0}(x)-t z^{d}=0
$$

where $\left(x_{1}, \ldots, x_{l}, z\right)$ are the homogeneous coordinates on $\mathbf{P}^{l}$ and $t$ is the coordinate on $\mathbf{C}$, and let $F: G \rightarrow \mathbf{C}$ be the projection. The composition with the inclusion $V \subset G$ (via $z \neq 0$ ) gives back $f$. The following properties are now easy to prove

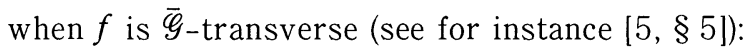


1. $G$ is smooth, as well as the closure in $G$ of every facet of the arrangement and $G-U$ is a hypersurface;

2. the restriction of $F$ to any such closure has only isolated singularities.

The stratification naturally associated with this arrangement in $G$ is a Whitney stratification, since the closure of every facet is a smooth submanifold of $G$. We can apply Proposition 2.2 to conclude.

The case of generic monodromy. Let $A_{\infty}$ the hyperplane at infinity in $\mathbf{P}^{l}$ and put $\mu_{\infty}=1 / \mu_{1} \cdots \mu_{p}$. Let $I=\{1, \ldots, p, \infty\}$. It is known that if the following condition $(\mathrm{H})$ on $\mu$ is satisfied, then $\operatorname{DR}(M(U))$ or equivalently $\boldsymbol{R} \Gamma\left(U^{\text {an }}, \mathscr{L}_{\mu}\right)$ has cohomology in degree $l$ only (see [11] for instance):

$$
\forall J \subset I \text { such that } \bigcap_{i \in J} A_{i} \neq \varnothing \text { one has } \prod_{i \in J} \mu_{i} \neq 1 \text {. }
$$

Moreover there exists a $\mu$ satisfying $(\mathrm{H})$ if and only if the arrangement does not come from an arangement in dimension less than $l$. We shall now prove an analogous result for $\mathcal{M}_{f}$. We introduce the following stronger condition on $\mu$ :

$$
\left\{\begin{array}{l}
\forall J \subset\{1, \ldots, p\} \text { such that } \bigcap_{i \in J} A_{i} \neq \varnothing \text { one has } \Pi_{i \in J} \mu_{i} \neq 1 \\
\forall J \subset I \text { containing } \infty \text { such that } \bigcap_{i \in J} A_{i} \neq \varnothing, \forall \nu \in \mathbf{Z}^{J} \\
\text { one has } \Pi_{i \in J} \mu_{i}^{\nu_{i}} \neq 1
\end{array}\right.
$$

As above, there exists such a $\mu$ if and only if the arrangement does not come from a lower dimensional arrangement. In practice it will be enough to satisfy the second part of $\left(\mathrm{H}^{\prime}\right)$ for a finite set of $\nu$.

Proposition 4.2. Assume that $\mu$ satisfies $\left(\mathrm{H}^{\prime}\right)$ and let $f$ be any polynomial on $V$. Then $\mathrm{DR}\left(\mathcal{M}_{f}(U)\right)$ has cohomology in degree $l$ at most.

Proof. Let $\tilde{\mathbf{P}}^{l} \rightarrow \mathbf{P}^{l}$ be the proper modification obtained by blowing up successively the facets of increasing dimension. The inverse image of $\cup_{\imath \in I} A_{\imath}$ is then a divisor with normal crossings $\tilde{D}$ (see [11]). Let $\pi: X \rightarrow \tilde{\mathbf{P}}^{l}$ be a proper modification on which $f$ extends as $F: X \rightarrow \mathbf{P}^{1}$ and for which the inverse image of $\cup_{i \in I} A_{i}$ and $F^{-1}(\infty)$ form a divisor with normal crossings. We can assume that $\pi$ is an isomorphism over the open set $\tilde{V}$ of $\tilde{\mathbf{P}}^{l}$ which lies over $V$. We can view $U$ as an open subset of $X$. In order to apply Proposition 3.1 to this situation, we need to 
compute the monodromy of $\mathscr{L}_{\mu}$ around the components of $Y-U$ (using the notation of Proposition 3.1). Indeed, because $Y-U$ is a divisor with normal crossings, the morphism $j_{!} \mathscr{L}_{\mu} \rightarrow \boldsymbol{R} j_{*} \mathscr{L}_{\mu}$ is an isomorphism if and only if the monodromy of $\mathscr{L}_{\mu}$ around each component of $Y-U$ is not equal to 1 . Using $\S \S 0.6$ and 0.7 we conclude that $j_{\dagger} \mathcal{M} \rightarrow j_{+} \mathcal{M}$ is an isomorphism under this condition, so the hypothesis (2) of 3.1 is satisfied for $\mathcal{M}$.

If the component intersects the inverse image of $V$, the computation is the same as the one in [11] and the first part of $\left(\mathrm{H}^{\prime}\right)$ implies that the monodromy is not equal to 1 .

If the component lies over $A_{\infty}$, the monodromy around it can be computed along a curve transverse to it. One can then compute it along the image of this curve in $\tilde{\mathbf{P}}^{l}$ and this is a local problem around a point on the divisor $\tilde{D}$. The second part of $\left(\mathrm{H}^{\prime}\right)$ implies that the monodromy cannnot be 1 .

\section{A local comparison theorem}

In this section, $X$ denotes a complex analytic manifold and $F: X \rightarrow \mathbf{C}$ is an analytic function. We are interested in the behaviour near the divisor $F^{-1}(0)$, so to compare with the statements in Section 1 , one has to replace $F$ with $1 / F$. Let $X^{*}=X-F^{-1}(0)$. Now $\mathscr{O}_{X}$ and $\mathscr{D}_{X}$ will denote the sheaves of analytic functions and analytic differential operators on $X$ and DR will denote the analytic de Rham functor. For a holonomic $\mathscr{D}_{X}$-module $\mathcal{M}$ we shall denote $\mathcal{M}\left[F^{-1}\right]$ the localized module along $F^{-1}(0)$ and $\mathcal{M}_{F}=\mathcal{M}\left[F^{-1}\right] \otimes e^{1 / F}$. These are known to be holonomic when $\mathcal{M}$ is so. We denote $\pi: \tilde{\mathbf{C}} \rightarrow \mathbf{C}$ the oriented real blowing-up of $\mathbf{C}$ at the origin (polar coordinates) and $\tilde{X}$ the fiber product $X \times_{\mathbf{C}} \tilde{\mathbf{C}}$. We define $I$ and $\tilde{X}_{I}$ as in the proof of Theorem $1.1\left(e^{-1 / t}\right.$ should be decreasing in the directions belonging to $I$ ). We consider also the inclusions $\eta: X^{*} \hookrightarrow X, \alpha: X^{*} \hookrightarrow \tilde{X}_{I}, \beta: \tilde{X}_{I} \hookrightarrow \tilde{X}$ and the projection $\pi: \tilde{X} \rightarrow X$.

Theorem 5.1. Let $\mathcal{M}$ be a regular holonomic $\mathscr{D}_{X}$-module. Then one has a quasi-isomorphism $\operatorname{DR}\left(\mathcal{M}_{F}\right) \stackrel{\sim}{\rightarrow} \boldsymbol{R} \pi_{*} \beta$ ! $\boldsymbol{R}_{*} \operatorname{DR}\left(\mathcal{M}_{\mid X^{*}}\right)$.

We shall deduce from this

COROLlary 5.2. The cone of the natural morphism

$$
\operatorname{DR}\left(\mu_{F}\right) \rightarrow \boldsymbol{R} \eta_{*} \operatorname{DR}\left(\mu_{\mid X^{*}}\right)=\operatorname{DR}\left(\mu\left[F^{-1}\right]\right)
$$

and the complex of nearby cycles $\phi_{F} \mathrm{DR}\left(\mathcal{M}\left[F^{-1}\right]\right)$ have the same characteristic func- 
tion.

Proof of Theorem 5.1. We may first assume that $F: X \rightarrow \mathbf{C}$ is isomorphic to a projection $Y \times \mathbf{C} \rightarrow \mathbf{C}:$ in order to do this we replace $X$ with $X \times \mathbf{C}, \tilde{X}$ with $X \times \tilde{\mathbf{C}}$ and $\boldsymbol{M}$ by the direct image of it by the graph embedding $X \hookrightarrow X \times \mathbf{C}$. For any $x \in Y$ there exists $\rho(x)>0$ such that, for $\rho<\rho(x)$, the direct image $F_{x, \rho+} M_{F}$ has coherent cohomologies and its germ at 0 does not depend on $\rho$, where $F_{x, \rho}$ is the restriction of $F$ to $B(x, \rho) \times D_{\delta(\rho)}$ and $B(x, \rho)$ is the open ball in $Y$ centered at $x$ and of radius $\rho, D_{\delta(\rho)}$ is the open disk of radius $\delta(\rho) \ll \rho$. This is a consequence of the coherence of the local Gauss-Manin system for $\mathcal{M}$ ([23, Theorem 9.4.1]).

We shall now consider the following diagram

$$
\begin{array}{rll}
\tilde{X}=Y \times \tilde{\mathbf{C}} \stackrel{\pi}{\rightarrow} & \mathrm{Y} \times \mathbf{C}=X \\
\tilde{F} \downarrow & & \downarrow F \\
\tilde{\mathbf{C}} & \stackrel{\varpi}{\rightarrow} & \mathbf{C}
\end{array}
$$

Let $\mathscr{A}_{\tilde{X}}^{\text {mod }}=\tilde{F}^{-1} \mathscr{A}^{\text {mod }} \bigotimes_{(\widetilde{F} \circ \varpi)^{-1} \mathscr{O}_{\mathbf{C}}} \pi^{-1} \mathscr{O}_{X}$. Because $\mathscr{A}^{\text {mod }}$ is (faithfully) flat over $\varpi^{-1} \mathscr{O}_{\mathbf{C}}$, one has

$$
\begin{aligned}
& \boldsymbol{R} \pi_{*} \mathscr{A}_{\tilde{X}}^{\bmod }=\boldsymbol{R}_{*}\left(\tilde{F}^{-1} \mathscr{A}^{\bmod } \underset{(\widetilde{F} \circ \varpi)^{-1} \mathscr{O}_{\mathbf{C}}}{\otimes} \pi^{-1} \mathscr{O}_{X}\right) \\
& =\boldsymbol{R} \pi_{*}\left(\tilde{F}^{-1} \mathscr{A}^{\mathrm{mod}} \underset{(\tilde{F} \circ \varpi)^{-1} \mathscr{O}_{\mathbf{C}}}{\boldsymbol{L}} \pi^{-1} \mathscr{O}_{X}\right) \text { (flatness) } \\
& =\left(\boldsymbol{R} \pi_{*} \tilde{F}^{-1} \mathscr{A}^{\mathrm{mod}}\right) \underset{F^{-1} \mathscr{O}_{\mathbf{C}}}{\stackrel{L}{L}} \mathscr{O}_{X} \text { (projection formula) } \\
& =\left(F^{-1} \boldsymbol{R} \varpi_{*} \mathscr{A}^{\text {mod }}\right) \underset{F^{-1} \mathscr{O}_{\mathbf{C}}}{\stackrel{L}{L}} \mathscr{O}_{X} \text { (see [10, Prop. 2.6.7]) } \\
& =\mathfrak{O}_{X}[*(Y \times\{0\})] \text {. }
\end{aligned}
$$

Define $\widetilde{\mathrm{DR}}^{\text {mod }}\left(M_{F}\right)=\mathscr{A}_{\tilde{X}}^{\text {mod }} \otimes_{\pi^{-1} \mathscr{O}_{X}} \pi^{-1} \mathrm{DR} M_{F}$. Then by the same argument one has $\mathrm{DR}\left(\mathcal{M}_{F}\right)=\boldsymbol{R} \pi_{*} \widetilde{\mathrm{DR}}^{\text {mod }}\left(\mathcal{M}_{F}\right)$. We want to show first that there exists a morphism

$$
\widetilde{\mathrm{DR}}^{\mathrm{mod}}\left(\mathcal{M}_{F}\right) \rightarrow \beta_{!} \boldsymbol{R} \alpha_{*} \mathrm{DR}\left(\mathcal{M}_{\mid X^{*}}\right)
$$

It is enough to show that $\gamma^{-1} \widetilde{\mathrm{DR}}^{\text {mod }}\left(\mathcal{M}_{F}\right)=0$, where $\gamma: \tilde{X} \rightarrow \tilde{X}_{I} \hookrightarrow \tilde{X}$ is the inclusion. This is a local problem on $\tilde{X}$, so one can prove it as in Section 1, using the coherence of the local Gauss-Manin system. One has to verify that

$$
\widetilde{\mathrm{DR}}^{\mathrm{mod}}\left(F_{x, \rho+} \mathcal{M}_{F}\right)=\boldsymbol{R} \tilde{F}_{x, \rho} * \overline{\mathrm{DR}}^{\mathrm{mod}}\left(M_{F}\right)
$$


which follows from the fact that the same is true for DR. In the same way one proves that this morphism is an isomorphism.

Proof of the corollary. As above we first reduce to the case where $F$ is the projection of a product $Y \times \mathbf{C}$. Denote $\partial \tilde{X}$ the restriction of $\tilde{X}$ over $S^{1}=\partial \tilde{\mathbf{C}}$. Let $\tilde{j}: X^{*} \hookrightarrow \tilde{X}$ and $\gamma: \tilde{X}-\tilde{X}_{I} \hookrightarrow \tilde{X}$ be the natural inclusions. The cone in the corollary is quasi-isomorphic to $\boldsymbol{R} \pi_{*}$ of the cone of

$$
\beta, \boldsymbol{R} \alpha_{*} \mathscr{F} \rightarrow \boldsymbol{R} \beta_{*} \boldsymbol{R} \alpha_{*} \mathscr{F}
$$

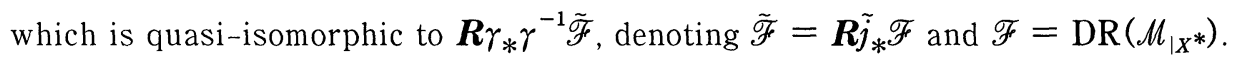

The computation is a local problem, so one can prove it by taking local direct images by $F_{x, \bar{\rho}}$, which is the restriction of $F$ to $\bar{B}(x, \rho) \times D_{\delta(\rho)}$. One is then reduced to prove the result in dimension one, and by an easy induction to the case where $\mathscr{F}$ is a local system, where the result is easy.

\section{REFERENCES}

[1] K. Aomoto, M. Kita, P. Orlik, H. Terao, Twisted de Rham cohomology groups of logarithmic forms, preprint (1994) to appear in Adv. in Math.

[2] A. Borel et al., Algebraic $\mathscr{D}$-modules, Perspectives in Math. vol. 2, Academic Press, Boston, 1987.

[ 3 ] A. Borel, Algebraic $\mathscr{D}$-modules, Chapters VI to IX in [2], 207-352.

[4] J. Briançon, Ph. Maisonobe, Sur la variété caractérsistique de systèmes différentiels irréguliers le long d'une hypersurface, C. R. Acad. Sci. Paris 320 (1995), $285-288$.

[5] S. A. Broughton, Milnor numbers and the topology of polynomial hypersurfaces, Invent. Math., 97 (1988), 217-241.

[6] J. L. Brylinski, (Co)homologie d'intersection et faisceaux pervers, in Séminaire Bourbaki, Astérisque, 92-93 (1982), 129-157.

[7] J. L. Brylinski, Transformations canoniques, dualité projective, in Géométrie et analyse microlocales, Astérisque, 140-141 (1986), 3-134.

[8] A. Dimca, M. Saito, On the cohomology of the general fiber of a polynomial map, Compositio Math., 85 (1993), 299-309.

[9] M. Kashiwara, On the maximally overdetermined systems of differential equations, Publ. R.I.M.S. Kyoto Univ., 10 (1975), 563-579.

[10] M. Kashiwara, P. Schapira, Sheaves on Manifolds, Grundlehren der mathematischen Wissenschaften 292, Springer-Verlag, Berlin, Heidelberg, 1990.

[11] T. Kohno, Homology of a local system on the complement of hyperplanes, Proc. Japan Acad., 62 (1986), 144-147.

[12] Lê D. T., Le concept de singularité isolée de fonction analytique, Advanced Studies in Pure Math., 8 (1986), 215-227.

[13] F. Loeser, C. Sabbah, Equations aux différences finies et déterminants d'intégrales de fonctions multiformes, Comment. Math. Helv., 66 (1991), 458-503. 
[14] B. Malgrange, Equations différentielles à coefficients polynomiaux, Progress in Math. vol. 96, Birkhaüser, Boston, 1991.

[15] B. Malgrange, letter to P. Deligne, April 30, 1991.

[16] Z. Mebkhout, Le formalisme des six opérations de Grothendieck pour les $\mathscr{D}$-modules cohérents, Hermann, Paris, 1989.

[17] Z. Mebkhout, Le théorème de comparaison entre cohomologies de de Rham d'une variété algébrique complexe et le théorème d'existence de Riemann, Publ. Math. I.H.E.S., 69 (1989), 47-89.

[18] Z. Mebkhout, Le théorème de positivité de l'irrégularité pour les $\mathscr{D}_{X}$-modules, in The Grothendieck Festschrift vol. III, Progress in Math., Birkhaüser, Boston, 88 (1990), 83-132.

[19] Z. Mebkhout, L. Narváez-Macarro, Démonstration géométrique du théorème de constructibilité, pages 248 to 253 in [16].

[20] Z. Mebkhout, L. Narváez-Macarro, Le théorème de constructiblilité de Kashiwara, in Eléments de la théorie des systèmes différentiels vol. 2, Hermann, Paris (1993), $47-98$.

[21] Z. Mebkhout, C. Sabbah, $\mathscr{D}$-modules et cycles évanescents, chapter III $\S 4$ in [16].

[22] M. Saito, letter to A. Dimca, April 30, 1991.

[23] M. Kashiwara, P. Schapira, Microlocal study of sheaves, Astérisque, 128 (1985).

URA 169 du C.N.R.S.

Centre de Mathématiques

Ecole Polytechnique

F-91128 Palaiseau cedex

France

e-mail:sabbah@math.polytechnique.fr 